\title{
Assessment of the structural validity of three foot and ankle specific patient-reported outcome measures
}

\section{Ponkilainen, Ville T.}

2020-02

Ponkilainen , V T , Tukiainen , E J , Uimonen , M M , Häkkinen , A H \& Repo , J P 2020 , ' Assessment of the structural validity of three foot and ankle specific patient-reported outcome measures ' , Foot and Ankle Surgery , vol. 26 , no. 2 , pp. 169-174 . https://doi.org/10.1016/j.fas.2019.01.00

http://hdl.handle.net/10138/327215

https://doi.org/10.1016/j.fas.2019.01.009

draft

Downloaded from Helda, University of Helsinki institutional repository.

This is an electronic reprint of the original article.

This reprint may differ from the original in pagination and typographic detail.

Please cite the original version. 


\title{
Assessment of the structural validity of three foot and ankle specific patient-reported outcome measures
}

\author{
Ville T. Ponkilainen ${ }^{\mathrm{a}, *}$, Erkki J. Tukiainen ${ }^{\mathrm{b}}$, Mikko M. Uimonen ${ }^{\mathrm{a}}$, Arja H. Häkkinen ${ }^{\mathrm{c}, \mathrm{d}}$, \\ Jussi P. Repo ${ }^{\mathrm{a}}$ \\ a Department of Surgery, Central Finland Central Hospital, Jyväskylä, Finland \\ ${ }^{\mathrm{b}}$ Department of Plastic Surgery, Central Hospital of Helsinki University, HUS, Helsinki, Finland \\ ${ }^{\mathrm{c}}$ Health Sciences, Faculty of Sport and Health Sciences, University of Jyväskylä, Jyväskylä, Finland \\ d Department of Physical Medicine, Central Finland Health Care District, Jyväskylä, Finland
}

\section{A R T I C L E IN F O}

\section{Article history:}

Received 20 October 2018

Accepted 21 January 2019

Available online $\mathrm{xxx}$

\section{Keywords:}

VAS-FA

LEFS

WOMAC

PROM

Validity

Outcome

Measures

\begin{abstract}
A B S T R A C T
Background: The structural validity of the Lower extremity functional scale (LEFS), the Visual analogue scale foot and ankle (VAS-FA), and the Western Ontario and McMaster Universities osteoarthritis index (WOMAC) has not been compared earlier in patients after foot and ankle surgery.

Methods: Altogether 165 previously operated patients completed the foot and ankle specific instruments, the 15D health-related quality of life (HRQoL) instrument, and general health (VAS).

Results: The LEFS, the VAS-FA and the WOMAC had slight differences in their measurement properties. The VAS-FA had the best targeting and coverage. All three foot and ankle measures accounted for mobility and usual activities when compared to the different aspects of generic HRQoL.

Conclusions: The LEFS, the VAS-FA and the WOMAC have relatively similar psychometric properties among foot and ankle patients, yet the VAS-FA provides the best targeting and coverage.
\end{abstract}

(c) 2019 European Foot and Ankle Society. Published by Elsevier Ltd. All rights reserved.

\section{Introduction}

The evaluation of the outcomes after surgical treatment with outcome rating scales has attracted more interest in recent years $[1,2]$. Patient-reported outcome measures (PROMs) are frequently used tools in evaluating the outcomes of surgical interventions of the foot and ankle [2,3]. PROMs are useful to assess the quality and outcomes of different treatments in clinical trials, and they provide possibilities for benchmarking [4].

The Lower extremity functional scale (LEFS) [5], the Visual analogue scale foot and ankle (VAS-FA) [6], and the Western Ontario and McMaster Universities osteoarthritis index (WOMAC) $[7,8]$ have been used to evaluate the outcomes in foot or ankle surgery [9,10]. The VAS-FA and the LEFS were originally developed to assess foot and ankle conditions, and they are both proven to be valid and reliable also in Finnish language $[5,6,11,12]$. The WOMAC was first introduced for patients with osteoarthritis of the hip and knee [7,8], yet it has been used and validated in patients with foot and ankle specific problems [13,14,16,36]. A

\footnotetext{
* Corresponding author at: Bachelor of Medicine, Department of Surgery, Central Finland Central Hospital, Keskussairaalantie 19, Jyväskylä, 40620, Finland.

E-mail address: ponkilainen.ville.t@student.uta.fi (V.T. Ponkilainen).
}

previous study by Pinsker et al. compared the LEFS, the WOMAC, the Foot Function Index (FFI), the Ankle Osteoarthritis Scale (AOS), Short Musculoskeletal Function Assessment (SMFA) and the patient-reported section of the American Orthopaedic Foot and Ankle Society Questionnaire (AOFAS) among patients with osteoarthritis of the ankle [17]. The study concluded that none of the PROMs captured patients' concerns properly, and there would be a further need for a valid instrument to assess foot and ankle specific problems [17].

At least 139 different assessment scales has been used in foot and ankle research [2]. Nonetheless, there have been a lack of consensus whether all used foot and ankle scales are valid and reliable [3]. The Consensus-based Standards for the selection of health status Measurement Instruments (COSMIN) includes a checklist to evaluate the quality of validation process of PROMs to clarify the basic standards which a PROM should fulfill [18]. As there has not been a consensus on which are the most suitable instruments for the foot and ankle patients [2], it is essential to assess existing instruments before developing new ones.

Current literature does not provide studies comparing the LEFS, the VAS-FA and the WOMAC instruments in patients with foot or ankle specific problems. We aimed to measure and compare the structural validity of these instruments in patients having undergone foot and/or ankle surgery. 


\section{Materials and methods}

The patients ( $n=212$ ) were identified from a database into which patients had been prospectively entered, or from an electronic database using the National Institute for Health and Welfare procedure codes which are based on the Nordic Medico-Statistical Committee (NOMESCO) classification (NHJ10, Ankle fracture osteosynthesis; NHU20 Removal of Implants from foot or ankle; NHG20 Tibiotalar joint fusion). The inclusion criteria of the study were at least 18 years of age, history of foot and/or ankle surgery, and full understanding of written language. The Regional Ethics Committee reviewed and approved the study protocol.

The participants were asked to fill in the LEFS, the VAS-FA, the WOMAC, and the 15D generic health-related quality of life (HRQoL) instrument. Furthermore, patients completed questionnaires about sociodemographic and clinical details, general health state on a VAS scale, and physical activity (Kasari FIT index [19]).

\subsection{Instruments}

\subsubsection{Lower extremity functional scale}

The LEFS has been developed for assessment of function of the lower extremity [5]. It is a PROM consisting of 20 items with 5 response categories awarding points between 0 and 4 . A higher score indicates better function of the foot and ankle [5].

The LEFS instrument has previously been validated for assessing the function of foot and ankle [5,11,20,21]. The internal consistency (Cronbach alpha 0.96), the construct validity, and the sensitivity to chance have been found to be high [5].

\subsubsection{Visual analogue scale foot and ankle}

The VAS-FA is a 20-item PROM developed and validated for the assessment of function and pain, and other symptoms of foot and ankle [12,22,23]. The instrument consists of 20 VAS-scaled items in three subscales: pain subscale of 4 items, Function subscale of 11 items, and Other complaints of 5 items [6]. Higher scores indicates better outcome [6]. The internal consistency of the subscales has been found high as the Cronbach alphas of the Function, Pain, and Other complaints subscales are $0.94,0.91$, and 0.81 , respectively [12]. Relative reliability has also been found high as the intra-class correlation coefficient (ICC) for total score and for the subscales are 0.97 and $0.95-0.97$, respectively [12].

\subsubsection{Western Ontario and McMaster Universities Osteoarthritis Index}

The WOMAC (version 3.0) is a VAS scaled 24-item PROM developed originally for measuring physical disability and symptoms in osteoarthritis of knee and hip [7,8]. Afterwards, it has been validated with osteoarthritis of foot and ankle patients $[17,24]$. It consists of three subscales: pain subscale of five items, Stiffness subscale of two items, and physical function subscale of 17 items with higher scores indicating higher amount of physical symptom and disability and vice versa $[7,8,25]$. The WOMAC has previously been validated using Rasch analysis, from which the findings indicated good fit of each subscale within the Rasch model. Factor analysis has also revealed a unidimensional construct of pain and physical subscales [25]. The internal consistency of pain, stiffness, and physical function subscales are high as the Cronbach alphas of the subscales are $0.82,0.80$, and 0.95 , respectively [25]. The WOMAC has previously been translated and validated in Finnish language [26,36].

\subsection{Statistical methods}

Clinical, sosiodemographic and questionnaire data are presented as means with standard deviations (SD), medians with interquartile ranges (IQR), 95\% confidence intervals (95\% CI), as counts with percentages, or as ranges. When $15 \%$ of patients scored the minimum or maximum points, a floor or ceiling effect was considered to be confirmed [27]. The WOMAC index scaling was reversed, so that it would be more explicit to compare it with the other instruments. Patients with complete data were included.

The Spearman correlation coefficients of the instruments were calculated to assess convergent validity. The correlations were represented as follows [28]: $0.00-0.30$ negligible, 0.30-0.50 low, $0.50-0.70$ moderate, $0.70-0.90$ high and $0.90-1.00$ very high correlation. Linearity between the instruments was assessed.

Linear regression analyses were used to identify the appropriate predictors of the LEFS, the VAS-FA, and the WOMAC. Age-, gender- and BMI-standardized regression coefficients (beta $\beta$ ) indicate how strongly each predictor variable influences the criterion (dependent) variable. The $\beta$ was measured in units of SD. Cohen reference values are 0.1 for small, 0.3 for moderate and 0.5 for strong correlations.

To conduct a regression analysis where all the reference outcome measures would be accounted, all the items of the 15D, the Kasari FIT -index and the VAS General health instruments were reduced to to factors using principal component (PC) analysis. A log transformation was applied to the continuous variables [29]. The PCs were chosen according to Kaiser criteria where a component was included if the eigenvalue was equal to 1 or higher [30]. Altogether four PCs fullfilled the Kaiser criteria. We analyzed the first PC as it explained the most (36.7\%) of the total variance. The PC was used to calculate how much variance the general health/ function-related variables would explain as a whole. One PC was used to eliminate multilinearity bias. Rotation local regression together with the LOESS curve with 95\% CIs was generated to illustrate the correlation of the PC with the LEFS, the VAS-FA, and the WOMAC instruments.

$\mathrm{R}$ (version 1.1.453) statistics software was used to perform the analyses. Results are interpreted and reported according to the Consensus-based Standards for the selection of health status Measurement Instruments (COSMIN) [18] and Strengthening the Reporting of Observational Studies in Epidemiology (STROBE) statement [31].

Table 1

Sociodemographic and clinical characteristics.

\begin{tabular}{ll}
\hline & $\mathrm{N}=165$ \\
\hline Female, n (\%) & $90(54.5)$ \\
Age, year, mean (SD) & $54.6(19.7)$ \\
BMI, mean (SD) & $27.3(4.9)$ \\
Education, n (\%) & $3(23.6)$ \\
Elementary school & $36(21.8)$ \\
Vocational school & $28(23.0)$ \\
High school & $47(28.5)$ \\
University & $73(44.2)$ \\
Working, n (\%) & $26(15.8)$ \\
Smoking, n (\%) & $70.5(59.0 ; 78.0)$ \\
LEFS score, median (IQR) & $0(0)$ \\
Minimum score, n (\%) & $29(176)$ \\
Maximum score, n (\%) & $83.2(56.1 ; 93.8)$ \\
VAS-FA score, median (IQR) & $0(0)$ \\
Minimum score, n (\%) & $4(2.4)$ \\
Maximum score, n (\%) & $91.4(75.4 ; 96.9)$ \\
WOMAC score, median (IQR) & 0 \\
Minimum score, n (\%) & $4(2.4)$ \\
Maximum score, n (\%) & $0.94(0.87 ; 0.97)$ \\
15D mean score, median (IQR) & $42.8(21.9)$ \\
Kasari FIT Index, mean (SD) & $14(4.0 ; 38.0)$ \\
VAS General health, median (IQR) &
\end{tabular}

All correlations were statistically significant $(P<0.001)$. 


\section{Results}

Altogether $165(77.8 \%)$ of the recruited patients $(n=212)$ provided valid questionnaires (Table 1 ). The questionnaires were completed averagely 4 years (range from 1 month to 10 years) after the surgery. Indication for surgery had been trauma $(n=156)$, infection $(n=6)$, tumor $(n=2)$ or osteoarthritis $(n=1)$. Location of the surgery was ankle $(n=133)$, hindfoot $(n=16)$, midfoot $(n=7)$, forefoot $(n=3)$, or multiple anatomical locations $(n=6)$. None of the three instruments had normally distributed scores (Fig. 1A-C). The ceiling effect was confirmed for the LEFS, as 29 (17.6\%) of the patients scored maximum points (Table 1 ).

\subsection{Correlations}

Fig. 2A-C describes the linear correlations for the LEFS, the VASFA and the WOMAC instruments. High correlations $(0.73-0.86 ; P<$ 0.001 ) indicated to linearity between all three instruments. The correlations between the LEFS, the VAS-FA and the WOMAC with the 15D dimensions are presented in the Table 2. There was a high correlation between the LEFS and the VAS-FA and the 15D Mobility
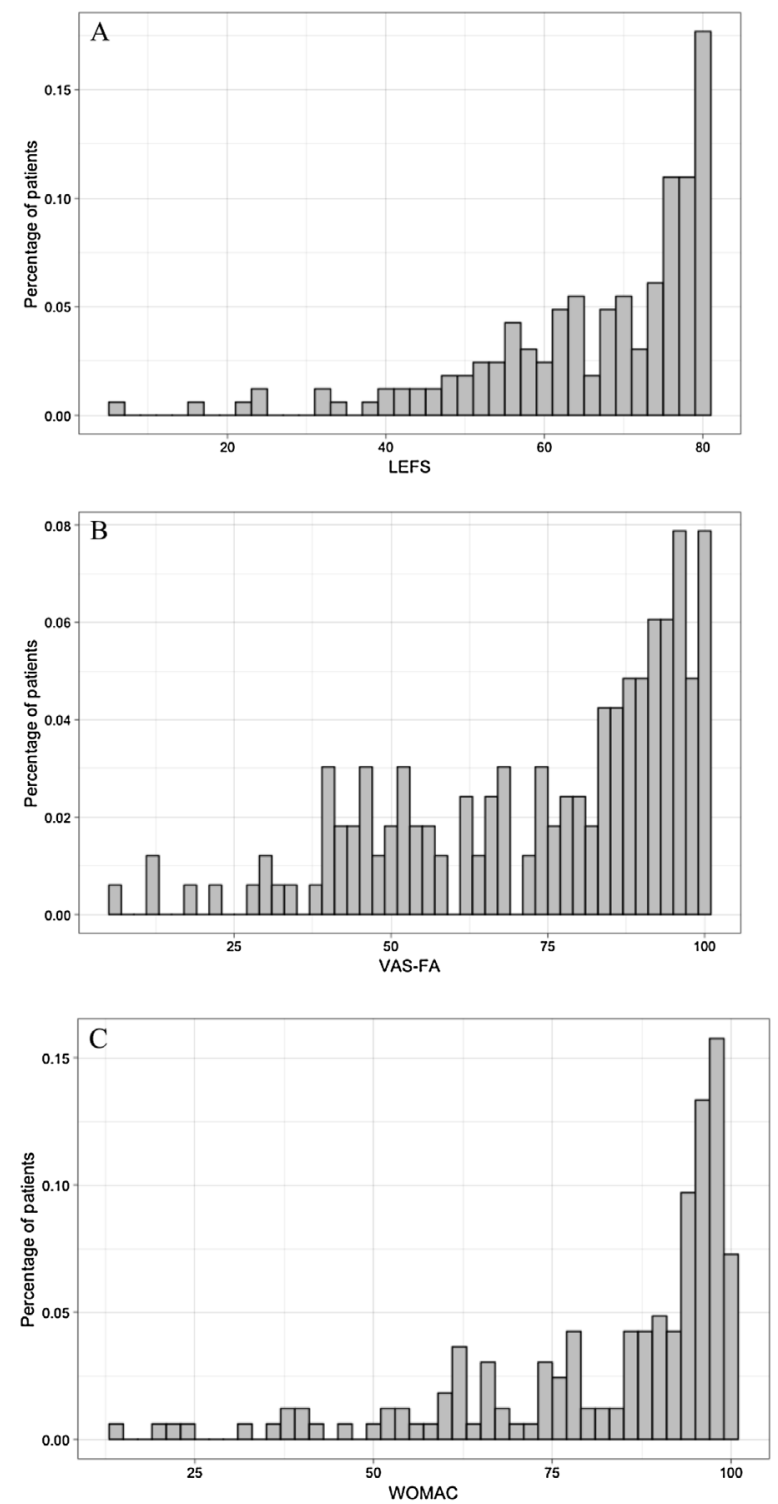

Fig. 1. A-C Distribution of the LEFS, the VAS-FA and the WOMAC total scores. dimension ( 0.74 and 0.70 , respectively). The correlations with the foot and ankle instruments were moderate between the 15D total score (0.60-0.68), and the 15D dimensions of Mobility (0.68-0.74), Usual Activities (0.50-0.61) and Discomfort (0.54-0.60), and the VAS General health instrument $(-0.53$ to -0.66$)$. All correlations were statistically significant $(P<0.001)$.

The LEFS, the VAS-FA and the WOMAC scores were compared with four states of general health (Fig. 3). The scores showed a gradual increase where general health was higher. The results for all instruments were relatively comparable.

Relationships between the instruments and the HRQoL (15D) were evaluated (Fig. 4). The relationships were strong with the 15D total score and the dimensions of Mobility $(\beta=0.68-0.72 ; P<0.001)$ and Usual activities $(\beta=0.54-0.69 ; P<0.001)$. Relationships with the VAS-FA, the WOMAC and the dimension of Discomfort and symptoms $(\beta=0.52-0.56 ; P<0.001)$ were strong, yet between LEFS and Discomfort and symptoms (15D) $(\beta=0.47 ; P<0.001)$ moderate. The LEFS had a strong relationship with the dimension of Vitality (15D) $(\beta=0.51 ; P<0.001)$, and the WOMAC and the VAS-FA scores had a moderate $(\beta=0.35-0.49 ; \mathrm{p}<0.001)$ relationship.
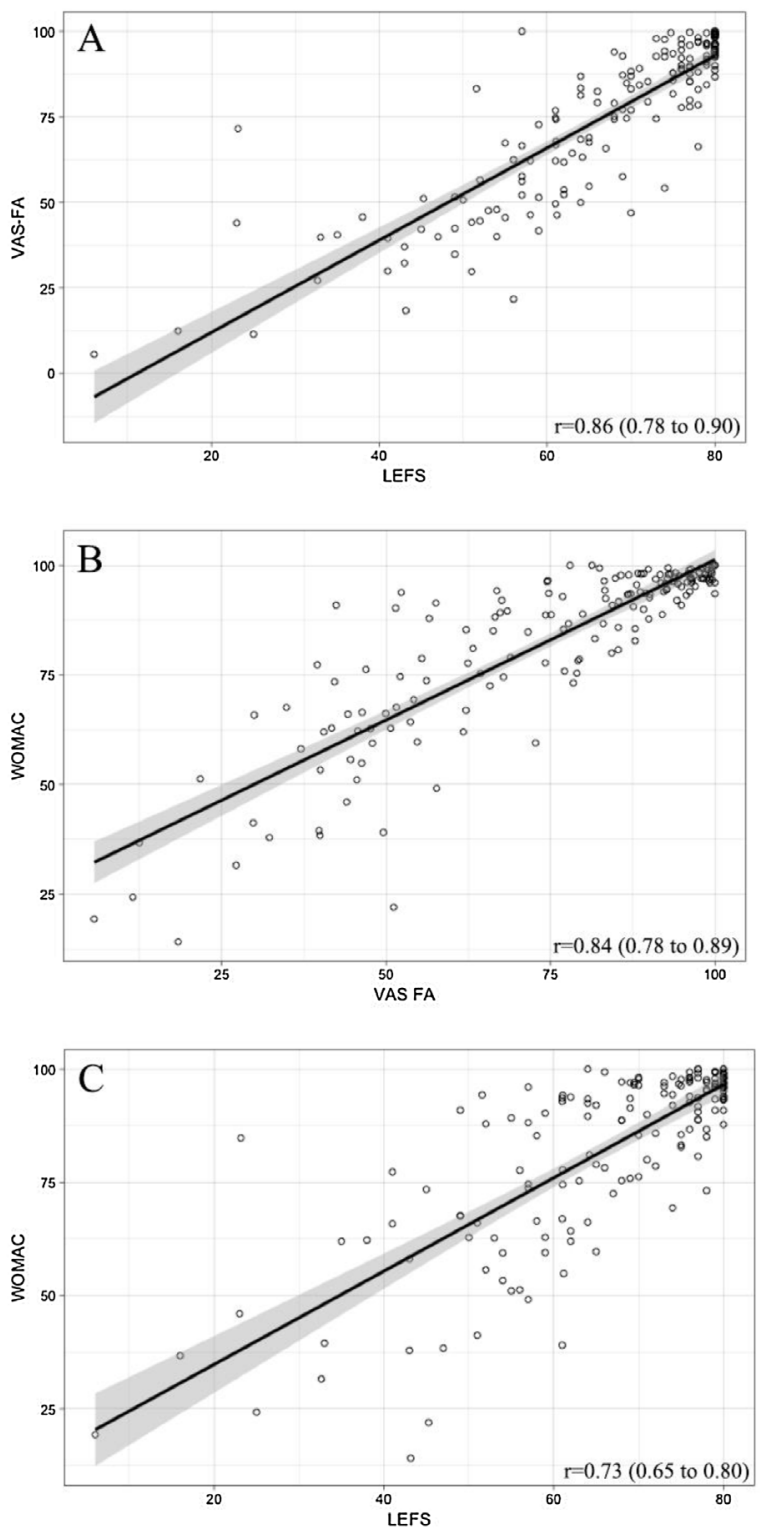

Fig. 2. A-C The Spearman correlations between the LEFS, the VAS-FA, WOMAC instruments. All correlations were statistically significant $(P<0.001)$. 
Table 2

Correlations between the LEFS, the VAS-FA, the WOMAC, and the dimensions of the 15D. Eating dimension was removed, since all patients scored maximum points.

\begin{tabular}{|c|c|c|c|}
\hline Variable & LEFS, r (95\% CI) & VAS-FA, r $(95 \% \mathrm{CI})$ & WOMAC, r (95\% CI) \\
\hline $15 \mathrm{D}$ total score & $0.67(0.57-0.74)$ & $0.68(0.58-0.76)$ & $0.60(0.49-0.69)$ \\
\hline \multicolumn{4}{|l|}{ 15D dimensions } \\
\hline Mobility & $0.74(0.66-0.81)$ & $0.70(0.60-0.77)$ & $0.68(0.58-0.75)$ \\
\hline Vision & $0.23(0.09-0.36)$ & $0.25(0.12-0.38)$ & $0.17(0.04-0.31)$ \\
\hline Hearing & $0.26(0.11-0.39)$ & $0.22(0.08-0.33)$ & $0.20(0.05-0.35)$ \\
\hline Breathing & $0.45(0.32-0.56)$ & $0.40(0.27-0.52)$ & $0.34(0.20-0.48)$ \\
\hline Sleeping & $0.22(0.07-0.36)$ & $0.21(0.05-0.35)$ & $0.17(0.02-0.32)$ \\
\hline Eating & - & - & - \\
\hline Speech & $0.21(0.11-0.32)$ & $0.18(0.07-0.28)$ & $0.15(0.06-0.24)$ \\
\hline Excretion & $0.23(0.08-0.38)$ & $0.18(0.01-0.33)$ & $0.11(0.03-0.27)$ \\
\hline Usual activities & $0.61(0.50-0.70)$ & $0.55(0.43-0.66)$ & $0.50(0.37-0.61)$ \\
\hline Mental function & $0.20(0.05-0.35)$ & $0.23(0.07-0.39)$ & $0.13(0.03-0.29)$ \\
\hline Discomfort & $0.54(0.41-0.64)$ & $0.60(0.49-0.69)$ & $0.58(0.46-0.68)$ \\
\hline Depression & $0.27(0.11-0.44)$ & $0.28(0.12-0.42)$ & $0.22(0.07-0.36)$ \\
\hline Distress & $0.20(0.05-0.35)$ & $0.26(0.11-0.39)$ & $0.22(0.07-0.36)$ \\
\hline Vitality & $0.49(0.34-0.61)$ & $0.49(0.35-0.60)$ & $0.41(0.27-0.55)$ \\
\hline Sexual activity & $0.40(0.26-0.52)$ & $0.38(0.23-0.51)$ & $0.33(0.19-0.46)$ \\
\hline FIT index & $0.44(0.31-0.57)$ & $0.35(0.20-0.48)$ & $0.31(0.16-0.44)$ \\
\hline VAS General health & $-0.66(-0.76$ to -0.55$)$ & $-0.61(-0.72$ to -0.49$)$ & $-0.53(-0.65$ to -0.39$)$ \\
\hline
\end{tabular}

All correlations were statistically significant $(P<0.001)$.

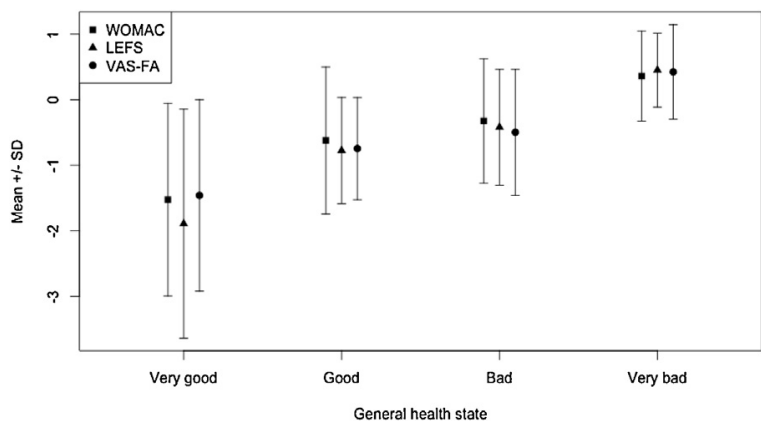

Fig. 3. The LEFS, the VAS-FA and the WOMAC scores compared with four states of general health. The whiskers show the SD.

Relationships between the reference instruments and the dimension of Breathing ( $\beta=0.35-0.49 ; P<0.001)$ were moderate.

Independent relationship of foot and ankle specific instruments were investigated against the principal component (Fig. 5A-C.). Scattering of scores was relatively similar for the LEFS, the VAS-FA and the WOMAC. However, the LEFS instrument had the most variance.

\section{Discussion}

To the best of our knowledge, this is the first study comparing the psychometric, structural validity of the LEFS, the VAS-FA and the WOMAC instruments among patients having undergone foot or ankle surgery. Main findings of our study were that these three foot and ankle PROMs have relatively similar psychometric properties when evaluating the outcomes of foot and ankle surgery. However, the LEFS instrument had a notable ceiling effect, indicating that the LEFS had the least properties of coverage and targeting for longterm outcome assessment of foot and ankle surgery. Considering coverage and targeting, the VAS-FA instrument seemed to have most suitable measurement properties in this patient sample.

Button and Pinney [3] published a meta-analysis on the PROMs used in the field of foot and ankle surgery. They concluded, that even though there are around 140 PROMs used among foot and ankle surgery, none of them were properly tested whether they fulfill the criteria for validity, reliability, and responsiveness, and being region-specific for foot and ankle [3]. According to their meta-analysis, the WOMAC was used in one study, and the LEFS was not used at all. Since their study, Richter [6] published a new instrument, the VAS-FA, to patch the lack of valid instruments in

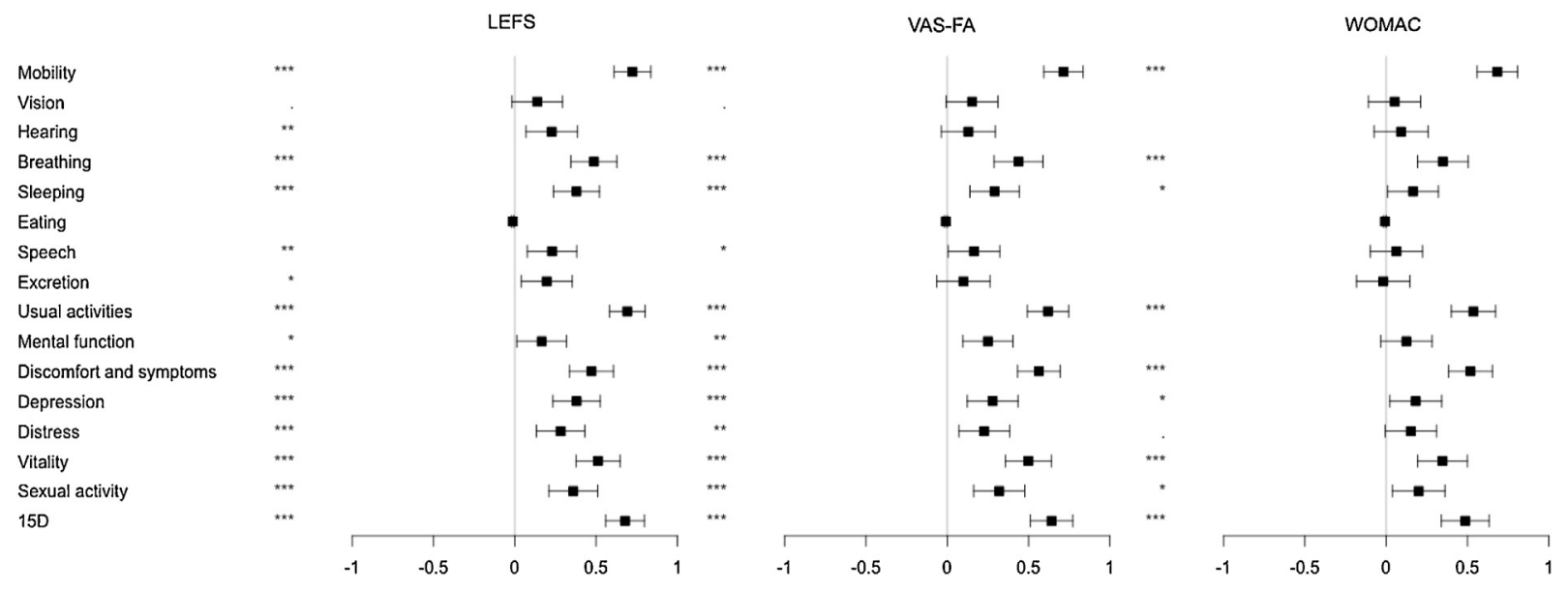

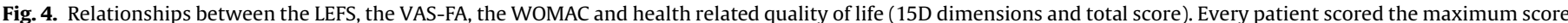

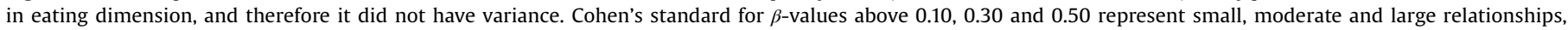
respectively. Boxes represent the mean scores (LEFS, VAS-FA, WOMAC). Whiskers show the $95 \%$ CIs. ${ }^{* * *} \mathrm{P}<0.001$. 

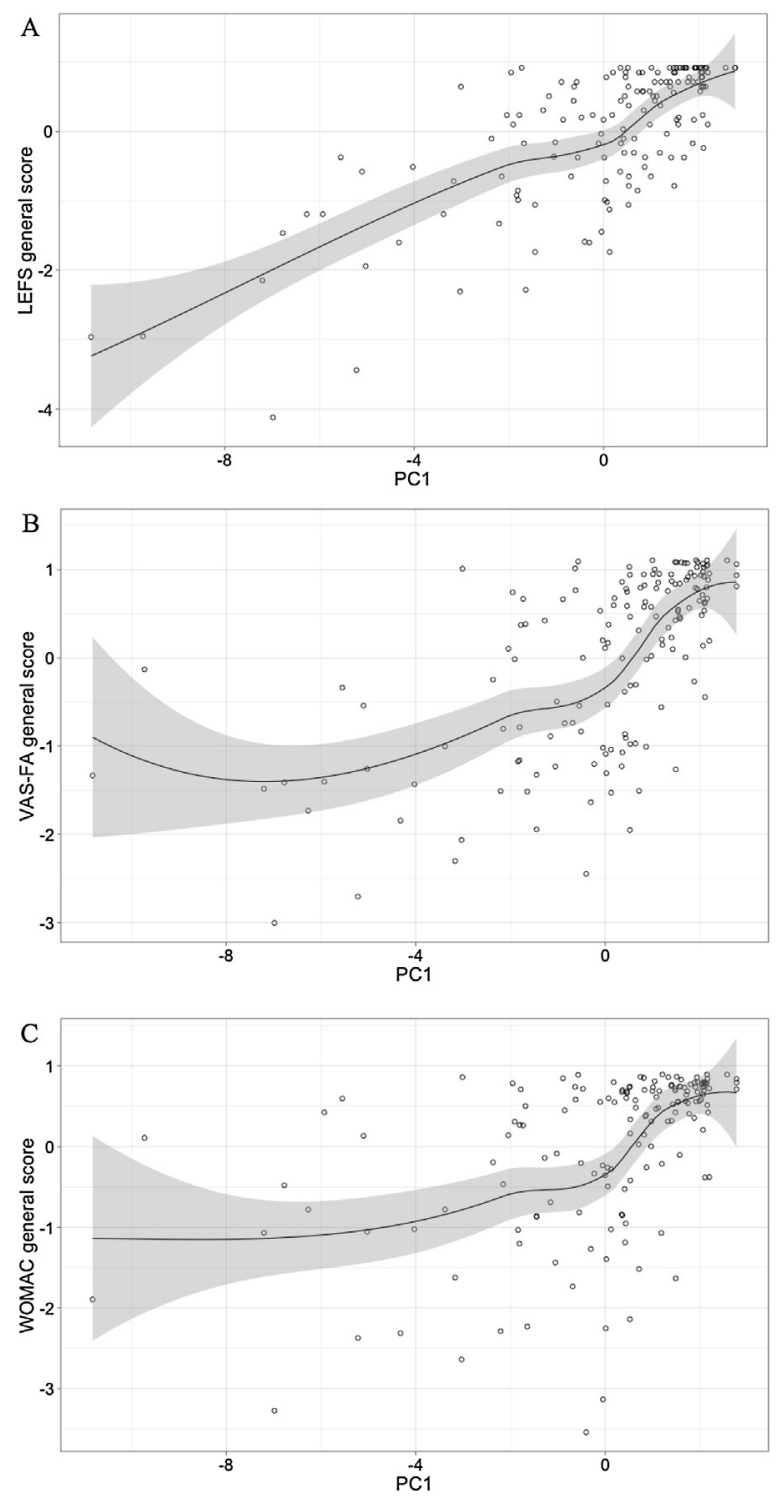

Fig. 5. A-C Relationship of the LEFS, the VAS-FA, and the WOMAC scaled scores (general score) with the first principal component (PC1). The LOESS curve shows the deterministic part of the variation in the data. Gray area around the curve describes the $95 \%$ CIs.

foot and ankle surgery. Nowadays, over twelve years after the meta-analysis, all three instruments have been validated properly $[6,25,32]$. Still, studies comparing all these three instruments have not been published.

In the present study, the correlations between the foot and ankle instruments and 15D dimensions did not show significant differences. All three foot and ankle instruments which were assessed in this study had a slightly higher correlation with the 15D dimensions related to physical function (Mobility, Usual activities and Discomfort and symptoms) than the other dimensions of the 15D instrument. When the scores of the foot and ankle instruments were compared with the four stages of general health, the instruments did not show significant differences. All the three foot and ankle instruments seem to evaluate the generic HRQoL of the patients quite similarly; yet they focus in physical functioning (Mobility, Usual activities, Discomfort and symptoms).

Pinsker et al. [17] compared six foot and ankle instruments, the LEFS, the WOMAC, the FFI, AOS, the AOFAS, and the SMFA, among patients with ankle arthritis. They concluded that all evaluated instruments were valid and reliable, and none of the foot and ankle specific scales offered advantages when comparing them to lower extremity instruments (the LEFS and the WOMAC). A new PROM called the EFAS score has recently been developed for foot and ankle patients by the score committee of the European Foot and Ankle Society (EFAS) [33]. This new instrument may provide further possibilities is assessing the treatment of foot and ankle diseases as it has been developed to overcome the weaknesses of other foot and ankle specific instruments [33].

In the present study, the VAS-FA foot and ankle specific instrument was compared with two lower extremity specific instruments (the LEFS and the WOMAC) in foot and ankle surgery. Although there were some differences between the scales, there seemed to be no real advantages using the instrument specifically designed for foot and ankle patients according to correlations, abilities to explain the principal component when it was compared to the lower extremity specific scales. In fact, the correlation was lower between the two lower extremity instruments than between the lower extremity instruments and the VAS-FA. The results of this study are indicating that the lower extremity specific instruments (the LEFS and the WOMAC) were measuring the same constructs as the VAS-FA which has been developed solely for foot and ankle assessment.

The strengths of our study were the representative study population and the use of properly validated and popularly used foot and ankle specific PROMs. The recruited study population contained patients with various indications for surgery, such as trauma, infection and tumors. The lower extremity specific LEFS [32] and the WOMAC [25] have been previously validated using the Rasch analysis [34], which can be considered as one of the golden standards in psychometric validation [35]. The weakness of this study was the lack of testing the responsiveness of these instruments. Furthermore, as the duration between the surgery and fulfilling the instruments was long (averagely 4 years), patients had high points on physical function and low level of pain in the evaluated instruments. When evaluating the validity of the PROMs, responsiveness is also an important measurement property to assess [18]. However, that was beyond the scope of the present study.

\section{Conclusions}

As a conclusion, the LEFS, the VAS-FA and the WOMAC have relatively comparable psychometric properties when evaluating the outcomes of foot or ankle surgery. However, the LEFS instrument has a notably higher ceiling effect compared to the other two measures indicating that the LEFS had the least suitable properties of coverage and targeting for long-term outcome assessment of foot and ankle surgery. Considering coverage and targeting, the results suggests that the VAS-FA instrument has the most suitable measurement properties in this patient sample.

\section{Conflict of interest statement}

All authors have completed the ICMJE uniform disclosure form at www.icmje.org/coi_disclosure.pdf and declare: no support from any organization for the submitted work. This work did not receive any funding.

\section{Acknowledgements}

This research received no specific grant from any funding agency in the public, commercial, or not-for-profit sectors. 


\section{References}

[1] Guyatt G, Cairns J, Churchill D, Cook D, Haynes B, Hirsh J, et al. Evidence-based medicine: a new approach to teaching the practice of medicine. JAMA 1992;268:2420.

[2] Hunt KJMD, Hurwit DBA. Use of patient-reported outcome measures in foot and ankle research. J Bone Joint Surg Am 2013;95:e118.

[3] Button G, Pinney S. A meta-analysis of outcome rating scales in foot and ankle surgery: is there a valid, reliable, and responsive system? Foot Ankle Int 2004;25:521.

[4] Nelson EC, Eftimovska E, Lind C, Hager A, Wasson JH, Lindblad S. Patient reported outcome measures in practice. BMJ 2015;350.

[5] Binkley JM, Stratford PW, Lott SA, Riddle DL. Network NAORR. The Lower extremity functional scale (LEFS): scale development, measurement properties, and clinical application. Phys Ther 1999;79:371.

[6] Richter M, Zech S, Geerling J, Frink M, Knobloch K, Krettek C. A new foot and ankle outcome score: questionnaire based, subjective, visual-analogue-scale, validated and computerized. Foot Ankle Surg 2006;12:191.

[7] Bellamy N, Buchanan WW, Goldsmith CH, Campbell J, Stitt LW. Validation study of WOMAC: a health status instrument for measuring clinically important patient relevant outcomes to antirheumatic drug therapy in patients with osteoarthritis of the hip or knee. J Rheumatol 1988;15:1833.

[8] Bellamy N. Validation study of WOMAC: a health status instrument for measuring clinically important patient-relevant outcomes following total hip or knee arthroplasty in osteoarthritis. J Orthop Rheumatol 1988;1:95.

[9] Rao S, Riskowski JL, Hannan MT. Musculoskeletal conditions of the foot and ankle: assessments and treatment options. Best Pract Res Clin Rheumatol 2012;26:345.

[10] Jia Y, Huang H, Gagnier JJ. A systematic review of measurement properties of patient-reported outcome measures for use in patients with foot or ankle diseases. Qual Life Res 2017;26:1969.

[11] Repo JP, Tukiainen EJ, Roine RP, Ilves O, Järvenpää S, Häkkinen A. Reliability and validity of the Finnish version of the Lower extremity functional scale (LEFS). Disabil Rehabil 2017;39:1228.

[12] Repo JP, Tukiainen EJ, Roine RP, Kautiainen H, Lindahl J, Ilves O, et al. Reliability and validity of the Finnish version of the visual analogue scale foot and ankle (VAS-FA). Foot and Ankle Surg 2018;24:474-80.

[13] Milner SA, Davis TRC, Muir KR, Greenwood DC, Doherty M. Long-term outcome after tibial Shaft fracture: is malunion important? J Bone Joint Surg 2002;84:971.

[14] Martin RL, Irrgang JJ, Lalonde KA, Conti S. Current concepts review: foot and ankle outcome instruments. Foot Ankle Int 2006;27:383.

[16] Stiegelmar R, McKee MD, Waddell JP, Schemitsch EH. Outcome of foot injuries in multiply injured patients. Orthop Clin North Am 2001;32:193.

[17] Pinsker E, Inrig T, Daniels TR, Warmington K, Beaton DE. Reliability and validity of 6 measures of pain, function, and disability for ankle arthroplasty and arthrodesis. Foot Ankle Int 2015;36:617.

[18] Mokkink LB, Terwee CB, Patrick DL, Alonso J, Stratford PW, Knol DL, et al. The COSMIN checklist for assessing the methodological quality of studies on measurement properties of health status measurement instruments: an international Delphi study. Qual Life Res 2010;19:539.
[19] Kasari D. The effects of exercise and fitness on serum lipids in college women. Unpublished Master's thesis. University of Montana; 1976. p. 46.

[20] Lin C-WC, Moseley AM, Refshauge KM, Bundy AC. The lower extremity functional scale has good clinimetric properties in people with ankle fracture. Phys Ther 2009;89:580.

[21] Pan S-L, Liang H-W, Hou W-H, Yeh T-S. Responsiveness of SF-36 and Lower extremity functional scale for assessing outcomes in traumatic injuries of lower extremities. Injury 2014;45:1759.

[22] Gur G, Turgut E, Dilek B, Baltaci G, Bek N, Yakut Y. Validity and reliability of visual analog scale foot and ankle: the Turkish version. J Foot Ankle Surg 2017;56:1213.

[23] Angthong C, Chernchujit B, Suntharapa T, Harnroongroj T. Visual analogue scale foot and ankle: validity and reliability of Thai version of the new outcome score in subjective form. J Med Assoc Thai 2011;94:952.

[24] Pinsker E, Daniels TR, Inrig T, Warmington K, Beaton DE. The ability of outcome questionnaires to capture patient concerns following ankle reconstruction. Foot Ankle Int 2013;34:65.

[25] Kersten P, White PJ, Tennant A. The visual analogue WOMAC 3.0 scale - internal validity and responsiveness of the VAS version. BMC Musculoskelet Disord 2010;11:80

[26] Soininen JV, Paavolainen PO, Gronblad MA, Kaapa EH. Validation study of a Finnish version of the Western Ontario and McMasters university osteoarthritis index. Hip Int 2008;18:108.

[27] McHorney CA, Tarlov AR. Individual-patient monitoring in clinical practice: are available health status surveys adequate? Qual Life Res 1995;4:293.

[28] Mukaka MM. A guide to appropriate use of correlation coefficient in medical research. Malawi Med J 2012;24:69.

[29] Venables WN, Ripley BD. Modern applied statistics with S-PLUS. New York: Springer-Verlag; 1997.

[30] Kaiser HF. The application of electronic computers to factor analysis. Educ Psychol Meas 1960;20:141.

[31] Von Elm E, Altman DG, Egger M, Pocock SJ, Gøtzsche PC, Vandenbroucke JP, et al. The strengthening the reporting of observational studies in epidemiology (STROBE) statement: guidelines for reporting observational studies. PLoS Med 2007; 4:e296.

[32] Repo J, Tukiainen E, Roine R, Sampo M, Sandelin H, Häkkinen A. Rasch analysis of the Lower extremity functional scale for foot and ankle patients. Disabil Rehabil 2018;1-7.

[33] Richter M, Agren P-H, Besse J-L, Cöster M, Kofoed H, Maffulli N, et al. EFAS score-development and validation by the score committee of the european foot and ankle society (EFAS). Foot Ankle Surg 2018;24:185-204.

[34] Rasch G. Studies in mathematical psychology: I. Probabilistic models for some intelligence and attainment tests. 1960.

[35] Panayides P, Robinson C, Tymms P. The assessment revolution that has passed England by: rasch measurement. Br Edu Res J 2010;36:611.

[36] Ponkilainen VT, Häkkinen AH, Uimonen MM, Tukiainen E, Sandelin H, Repo JP. Validation of The Western Ontario and McMaster Universities Osteoarthritis Index (WOMAC) in patients having undergone ankle fracture surgery. J Foot Ankle Surg 2019 (in press). 